\title{
Cobertura en suelos con procesos acelerados de erosión
}

\section{Soil cover with accelerated erosion processes}

\author{
Beltrán Guarnizo Laura ${ }^{1}$ y Pérez López Otoniel ${ }^{2}$ \\ ${ }^{1}$ I. A. Universidad de los Llanos y \\ 2.. A. Investigador Profesional Asociado, CORPOICA \\ operez@corpoica.org.co
}

Recibido 11 de Abril 2014, Aceptado 17 de Octubre 2014

\section{RESUMEN}

El objetivo del estudio fue evaluar la introducción y la persistencia de cobertura vegetal en suelos con procesos acelerados de erosión, en Puerto Gaitán, Meta, donde se evaluaron diversas especies y prácticas de manejo del suelo. La fertilización de las parcelas experimentales se realizó con una saturación de bases de 35 a $50 \%$ y niveles de fósforo entre 5 y 10 ppm. Los correctivos utilizados fueron cal dolomita, roca fosfórica, abono paz del rio y oxido de magnesio. Las actividades estratégicas que se plantearon incluyeron: evaluación de herbáceas (gramíneas y leguminosas) solas o en combinación con arbóreas y arbustivas; evaluación de prácticas, sistemas de labranza y transferencia de tecnología. En suelo se valoró durante tres años la dinámica de características químicas: $\mathrm{pH}$, materia orgánica, $\mathrm{P}, \mathrm{Ca}, \mathrm{Mg}, \mathrm{K}, \mathrm{S}, \mathrm{Al}, \mathrm{Fe}, \mathrm{Cu}, \mathrm{Mn}, \mathrm{Zn}$ y $\mathrm{B}$ en varias profundidades (0-10, 0-20 cm o según los horizontes dominantes), durante la época de máxima y mínima precipitación. En las especies vegetales se evaluó emergencia (\%); altura $(\mathrm{cm})$; cobertura (\%); ataque de plagas y enfermedades; distribución y desarrollo radicular de especies con sobresaliente producción y calidad de biomasa verde seca y hojarasca. Al finalizar el establecimiento $B$. dictyoneura, $B$. decumbens, $D$. ovalifolium y $B$. humidicola mostraron la mayor cobertura del suelo $(P<0.05)$ con valores de 93.3, 90.0. 88.3 y 76.7\%, respectivamente en comparación con Stylosanthes capitata (28.3\%) y Vitiveria zizanoides (36.7\%). Aunque $B$ dictyoneura obtuvo una cobertura del $93.3 \%$ su establecimiento fue más lento en comparación con $B$. decumbens y $B$. humidicola fue más lento; no obstante, una 
vez alcanzado su óptimo crecimiento, su cobertura fue eficiente por sus estolones y rizomas, lo que le permitió de igual forma al $B$. humidicola obtener una cobertura del $77 \%$, con buen desarrollo vigoroso y agresivo.

Palabras clave: Vegetación, gramíneas, biomasa, recuperación.

\begin{abstract}
The aim of the study was to evaluate the introduction and persistence in soil mulched with accelerated erosion in Puerto Gaitan, Meta, where various species and soil management practices was evaluated. Fertilization of the experimental plots was performed with a base saturation of $35-50 \%$ and phosphorus levels between 5 and 10 ppm. Corrective used were lime dolomite, phosphate rock, fertilizer Peace River and magnesium oxide. The strategic activities that were raised included: assessment of herbaceous (grasses and legumes) alone or in combination with trees and bushes; assessment practices, tillage systems and technology transfer. In soil was assessed for three years dynamics chemical characteristics: $\mathrm{pH}$, organic matter, $\mathrm{P}, \mathrm{Ca}, \mathrm{Mg}, \mathrm{K}, \mathrm{S}, \mathrm{Al}, \mathrm{Fe}, \mathrm{Cu}, \mathrm{Mn}, \mathrm{Zn}$ and $\mathrm{B}$ at various depths $(0-10,0-20 \mathrm{~cm}$ or according to the dominant horizons), during the time of maximum and minimum precipitation. Emergence (\%); height (cm); coverage (\%); pest and disease attack; distribution and root development of species with outstanding production and quality of dry green biomass and litter was evaluated in plant species. At the end of the establishment $B$. dictyoneura, $B$. decumbens, $B$. humidicola and $D$. ovalifolium showed the highest soil cover $(\mathrm{P}<0.05)$ with values of $93.3,90.0 .88 .3$ and $76.7 \%$ respectively compared with Stylosanthes capitata (28.3\%) and Vitiveria zizanoides (36.7\%). While $B$. dyctioneura obtained $93.3 \%$ coverage of its establishment was slower compared with $B$. decumbens and $B$. humidicola was slower; however, once you reach your optimum growth was efficient coverage for their stolons and rhizomes, allowing him equally to $B$. humidicola get coverage of $77 \%$, with good vigorous and aggressive development.
\end{abstract}

Keywords: Vegetation, grasses, biomass, recovery. 


\section{RESUMO}

O objetivo do estudo foi avaliar a introdução e persistência no solo mulched com erosão acelerada em Puerto Gaitán, Meta, onde várias espécies e práticas de manejo do solo foram avaliados. Fertilização das parcelas experimentais foi realizada com a saturação por bases de 35-50\% e fósforo níveis entre 5 e 10 ppm. Corretiva foram usadas calcário dolomítico, fosfato, fertilizantes Peace River e óxido de magnésio. As atividades estratégicas que foram levantadas foram: avaliação de herbáceas (gramíneas e leguminosas), isoladamente ou em combinação com árvores e arbustos; práticas de avaliação, sistemas de preparo e transferência de tecnologia. Em solo foi avaliada por três anos de dinâmica características químicas: $\mathrm{pH}$, matéria orgânica, $\mathrm{P}, \mathrm{Ca}, \mathrm{Mg}, \mathrm{K}, \mathrm{S}, \mathrm{Al}, \mathrm{Fe}, \mathrm{Cu}, \mathrm{Mn}, \mathrm{Zn}$ e $B$ em várias profundidades $(0-10,0-20 \mathrm{~cm}$ ou de acordo com os horizontes dominantes), durante os tempos de precipitação máxima e mínima. A emergência (\%); altura (cm); cobertura (\%); ataque de pragas e doenças; distribuição e desenvolvimento de raízes de espécies com excelente produção e qualidade de biomassa verde seca e serapilheira foi avaliada em espécies de plantas. No final do estabelecimento $B$. dictyoneura, $B$. decumbens, $B$. humidicola e $D$. ovalifolium apresentou a maior cobertura do solo $(P<0.05)$ com valores de 93,3, 90.0. 88,3 e $76,7 \%$ respectivamente, em comparação com Stylosanthes capitata $(28,3 \%)$ e Vitiveria zizanoides $(36,7 \%)$. Enquanto $B$ dictyoneura obteve uma cobertura de $93,3 \%$ do seu estabelecimento foi mais lento em comparação com $B$. decumbens e $B$. humidicola foi mais lento, no entanto, uma vez que atingiu o seu crescimento óptimo foi a cobertura eficiente para seus estolões e rizomas, permitindo-lhe igualmente a $B$. humidicola obter uma cobertura.

Palavras-chave: Vegetação, gramíneas, biomassa, recuperação.

\section{INTRODUCCIÓN}

El suelo es un recurso vital para el hombre debido a la relación de dependencia entre ambos puesto que, en él encuentran soporte gran número de actividades productivas de los sectores de alimentos, industria y vivienda, entre otros. En el 
caso de la aplicación directa del suelo a actividades productivas, como la agricultura o el pastoreo, se requiere de sustancias nutritivas para el desarrollo de las plantas, la mayor o menor concentración y disponibilidad de nutrientes refleja el nivel de fertilidad de un suelo, sin embargo, es en su capa más superficial donde se concentra la mayor fertilidad, que en ocasiones alcanza escasamente unos pocos centímetros de espesor, lo cual plantea serias implicaciones dado el desbalance existente entre las tasas de formación del suelo y las de erosión que pueden alcanzarse bajo determinadas condiciones, así, un centímetro de suelo puede tardar en su formación cientos, e incluso miles de años, en tanto que en un aguacero o por efecto de la acción del viento, éste puede perderse en pocos segundos (Burbano, 2007).

Los sistemas de manejo tecnológico y de apropiación del suelo para las actividades agrícolas, pecuarias y forestales, entre otras, han ido muchas veces en detrimento de este recurso, ocasionando una mínima posibilidad de sostenibilidad en el tiempo. El proceso comienza con la ruptura de un equilibrio que se ha dado a través de una interacción ininterrumpida (a excepción de fenómenos igualmente naturales) entre el medio físico y el medio biótico, con la remoción de la vegetación del suelo para dar entrada a otras formas vegetales, orientadas hacia la producción; luego se rompe la superficie de los terrenos y se somete el suelo a un laboreo periódico con elementos de labranza, apareciendo el fenómeno erosivo y marcándose de esta forma un punto de quiebre frente al criterio de sostenibilidad, dadas las significativas diferencias entre la tasa de formación y la tasa de pérdida de suelo mencionadas. Esta descripción corresponde en esencia a la génesis del fenómeno entonces vigente de forma casi exclusiva, hoy día, a las pérdidas de suelo imputables a la aplicación de tierras a la agricultura y pastoreo, deben sumarse aquellas originadas del adelanto de proyectos de desarrollo (carreteras, ferrocarriles, aeropuertos, grandes presas y otros), cuya inadecuada gestión en el pasado generó importantes impactos ambientales en términos de pérdidas de suelo, y en algunos casos los sigue generando actualmente (León, 2001). 
Se destacan tres tipos de proyectos de relevancia en la generación del problema como son las urbanizaciones, puesto que su proceso de construcción aporta importantes cantidades de sedimentos, dado que las actividades como la remoción de las coberturas vegetales, la excavación, y la explanación, entre otras, dejan grandes masas de suelo expuestas a la acción de la lluvia, provocando su desprendimiento y arrastre o transporte; una vez se tienen áreas urbanizadas completamente desarrolladas, la producción de sedimentos es, por lo general, mínima. En el proceso de su construcción de carreteras también pueden darse altas tasas de erosión al ser removidos los materiales vegetales, dejando el suelo descubierto. En tercer lugar, las obras de control en ríos y corrientes: cambios en la dirección del flujo o aumento en la profundidad, duración y velocidad de éste, pueden promover procesos erosivos; se citan entre otros: el alineamiento de canales serpenteados que aumenta la pendiente y la velocidad de flujo; la restricción de la sección transversal del canal, aumentándose la velocidad y profundidad del flujo; y la construcción de presas que influye en la estabilidad del canal aguas arriba y aguas abajo (Castañeda y Contreras, 2003).

\section{METODOLOGÍA}

Como el propósito de la siembra de gramíneas y leguminosas para la recuperación y persistencia de la cobertura vegetal y de esta evitar los procesos acelerados de erosión del suelo, se evaluaron diversas especies y prácticas de manejo del suelo. La fertilización de las parcelas experimentales se realizó con una saturación de bases entre 35 a 50\% y niveles de fósforo entre 5 a 10 ppm. Los correctivos que se utilizaron son enmiendas comerciales disponibles en el mercado como cal dolomita, roca fosfórica, abono paz del río y oxido de magnesio. Las actividades estratégicas que se plantearon incluyeron: evaluación de materiales herbáceos (gramíneos y leguminosos) solos o en combinación con arbóreas y arbustivas; evaluación de prácticas y sistemas de labranza; y transferencia de tecnología. 


\section{Establecimiento de parcelas experimentales}

Evaluación de materiales herbáceos. Incluyó el establecimiento de materiales herbáceos de gramíneas (Brachiaria decumbens, Brachiaria dictyoneura, Brachiaria humidicola, Andropogon gayanus y Vitiveria zizanioides) y leguminosas (Pueraria phaseoloides, Stylosanthes capitata y Desmodium ovalifolium) en suelo descubierto. Los tratamientos se distribuyeron en un diseño al azar con tres repeticiones, el tamaño de parcelas para las herbáceas fue $2 \mathrm{~m} \times 5 \mathrm{~m}\left(10 \mathrm{~m}^{2}\right)$. La preparación del terreno consistió en dos pases de rastra, para la fertilización de establecimiento se aplicó en $\mathrm{kg} / \mathrm{ha}$ una mezcla de: cal dolomita (1500), roca fosfórica (500), abono paz del rio (500) y yeso agrícola (400). Se realizó la siembra de $B$. humidicola, $B$. decumbens, $B$. dictyoneura, $S$. capitata, $D$. ovalifolium y $P$. phaseoloides con semilla cariópside en surcos distanciados a $30 \mathrm{~cm}$, mientras que con material vegetativo (cepas) se establecieron las parcelas de $V$. zizanioides y A. gayanus a una distancia de $40 \mathrm{~cm}$ entre plantas y entre surcos. Las parcelas fueron fertilizadas con una mezcla en $\mathrm{kg} / \mathrm{ha} 50$ de urea, 25 de cloruro de potasio (KCl) y 25 de fosfato diamónico (DAP).

Evaluación de materiales arbustivos: Incluyó el establecimiento de arbustivos (Cratylia argentea, Codariocalyx gyroides y Ficus macrophylla) en suelo descubierto. Los tratamientos se distribuyeron en un diseño al azar con tres repeticiones. El tamaño de parcelas fue de $3 \mathrm{~m} \times 10 \mathrm{~m}\left(30 \mathrm{~m}^{2}\right)$. La preparación del terreno consistió en dos pases rastra y un pase de cincel rígido; para la fertilización de establecimiento se aplicó e incorporó una mezcla igual a la aplicada en las gramíneas. Se realizó la siembra de las semillas a una distancia de $1 \mathrm{~m} \times 1$ $\mathrm{m}$ entre plantas y entre surcos.

Evaluación de materiales arbóreos: Incluyó el establecimiento de materiales arbóreos (Acacia mangium, Anacardium occidentale, Piptadenia opacifolia, Eucalipthus pellita, Simarouba amara y Pinus caribeae) en suelo descubierto. Los tratamientos se distribuyeron en un diseño al azar con tres repeticiones. El tamaño de parcelas para las arbóreas es de $10 \mathrm{~m}$ X $15 \mathrm{~m}$ (150 m²). La preparación del terreno y aplicación de fertilizantes fue igual a la suministrada a herbáceas y 
arbustivas. Se realizó la siembra a una distancia de $5 \mathrm{~m} \times 5 \mathrm{~m}$ entre plantas y entre surcos. Para la fertilización de mantenimiento se aplicó una mezcla de 95 g/planta compuesta por: Urea 30, $\mathrm{KCl} 30, \mathrm{DAP} 20, \mathrm{MgSO}_{4} 10, \mathrm{CuSO}_{4} 2$, Boro 3 y $\mathrm{ZnSO}_{4} 3 \mathrm{~g}$ respectivamente.

\section{Evaluación de prácticas de preparación y manejo en suelos descubiertos} para establecimiento de especies herbáceas. Se sembró en asociación $B$. decumbens y $B$. dictyoneura, los tratamientos se distribuyeron al azar en un diseño de franjas divididas con tres repeticiones, en donde el tamaño de parcelas fue de $5 \mathrm{~m} \times 10 \mathrm{~m}\left(50 \mathrm{~m}^{2}\right)$ y consistieron en: 1) un pase de rastra sin correctivos; 2) dos pases de rastra sin correctivos; 3) dos pases de rastra con correctivos; y 4) un pase de rastra, uno de cincel con correctivos.

Evaluación de fuentes fosforadas en suelos descubiertos para establecimiento de especies herbáceas. Se evaluó el efecto de diferentes fuentes de fosforo, mediante la aplicación de insumos fosforados como roca fosfórica, abono paz del rio y DAP para el establecimiento de la mezcla de semillas cariópside $B$. decumbens $+B$. dictyoneura en condiciones de suelo de la Altillanura disectada. Se utilizó un diseño completo al azar con tres repeticiones, parcelas de tamaño $4 \mathrm{~m} \times 5 \mathrm{~m}\left(20 \mathrm{~m}^{2}\right)$. Los tratamientos fueron: 1) cal dolomita 1,5 t/ha con abono paz del rio (46 kg/ha $\left.\mathrm{P}_{2} \mathrm{O}_{5}\right) ; 2$ ) cal dolomita $1,5 \mathrm{t} / \mathrm{ha}$ con roca fosfórica (46 kg/ha $\left.\mathrm{P}_{2} \mathrm{O}_{5}\right)$; y 3 ) cal dolomita 1,5 ton/ha con DAP (46 kg/ha $\left.\mathrm{P}_{2} \mathrm{O}_{5}\right)$. El lote se mecanizó con dos pases de rastra, y se aplicó una fertilización basal conformada por cal dolomita (1500 kg/ha) con yeso agrícola (400 kg/ha).

En el suelo se evaluó dinámica de características químicas: pH, materia orgánica, $\mathrm{P}, \mathrm{Ca}, \mathrm{Mg}, \mathrm{K}, \mathrm{S}, \mathrm{Al}, \mathrm{Fe}, \mathrm{Cu}, \mathrm{Mn}, \mathrm{Zn}$ y $\mathrm{B}$ durante un periodo de 3 años en varias profundidades ( 0 a 10, 0 a $20 \mathrm{~cm}$ o según los horizontes dominantes) al inicio y al final del proyecto; y características físicas: densidad aparente, porosidad, retención de humedad, resistencia a la penetración a tres profundidades $(0$ a 10, 0 a $20 \mathrm{~cm}$ o según los horizontes dominantes); biología de suelos: (carbono y nitrógeno de la biomasa microbiana) en los primeros $10 \mathrm{~cm}$ de profundidad; y especies vegetales durante la época de máxima y mínima precipitación. 
En las especies vegetales se evaluó emergencia (\%), altura $(\mathrm{cm})$, cobertura (\%), ataque de plagas y enfermedades, producción de biomasa verde seca y hojarasca como: proteína cruda, materia orgánica, FDN, digestibilidad, $\mathrm{P}$ y $\mathrm{Ca}$. En las arbóreas y arbustivas se evaluó sobrevivencia $(\%)$, altura de planta $(\mathrm{cm})$, diámetro a la altura del pecho $(\mathrm{cm})$, ecuaciones de crecimiento, índice de sitio para cada especie y cada tratamiento teniendo en cuenta que este parámetro relaciona edad con crecimiento.

\section{RESULTADOS Y DISCUSIÓN}

En un área representativa de la altillanura disectada se establecieron cinco ensayos correspondientes a dos fases de evaluación; la primera fue la selección de materiales vegetales (herbáceos, arbóreos y arbustivos) y la segunda la evaluación de prácticas de manejo agronómico (mecanización de suelos y fuentes de fosforo). La información registrada corresponde a la fase de establecimiento de las especies forrajeras en un periodo aproximado de 120 días después de la siembra.

\section{Análisis de suelos en áreas experimentales}

Antes de iniciar la siembra de las parcelas experimentales se tomaron muestras de suelo a dos profundidades ( 0 a 10 y 10 a $20 \mathrm{~cm}$ ) en tres áreas representativas. Los resultados indican una fuerte acidez ( $\mathrm{pH} 4.6$ a 5.45), bajos contenidos de materia orgánica ( 0.73 a 2.1), y alta proporción de hierro, baja saturación de bases intercambiables como calcio, magnesio, potasio y fósforo (Tabla 1).

\section{Evaluación de materiales herbáceos}

1. Emergencia y sobrevivencia de plantas. En general los materiales mostraron buena emergencia y sobrevivencia en campo, S. capitata presentó una emergencia lenta y baja población de plántulas requiriendo una resiembra. Durante la fase de establecimiento (120 días), sobresalió $(\mathrm{P}<0.05)$ la leguminosa Desmodium ovalifolium, por su alta población de plantas en campo. Entre las gramíneas el pasto $B$. decumbens presentó una mayor 
capacidad germinativa y viabilidad de la semilla. $B$. dictyoneura y $B$. humidicola a pesar de presentar dormancia en su semilla mostraron una buena población de plantas durante los estados iniciales. Las especies establecidas con material vegetativo $A$. gayanus y $V$. zizanioides mostraron una excelente sobrevivencia al mantener la totalidad de las plantas con buen vigor y desarrollo de macollas.

Tabla 1. Características químicas de los suelos (Campo Rubiales 2010)

\begin{tabular}{|c|c|c|c|c|c|c|}
\hline \multirow{2}{*}{$\begin{array}{c}\text { Parámetro } \\
\text { Profundidad }(\mathrm{cm})\end{array}$} & \multicolumn{2}{|c|}{ Plintita expuesta } & \multicolumn{2}{|c|}{ Arbóreas } & \multicolumn{2}{|c|}{$\begin{array}{c}\text { Suelo } \\
\text { descubierto }\end{array}$} \\
\hline & 10 & 20 & 10 & 20 & 10 & 20 \\
\hline $\mathrm{pH}$ & 4.97 & 5.45 & 4.80 & 5.20 & 4.90 & 4.60 \\
\hline $\mathrm{MO}(\%)$ & 0.73 & 0.45 & 1.90 & 1.03 & 1.13 & 2.10 \\
\hline CICE meq/100 g & 1.10 & 0.85 & 2.13 & 1.88 & 0.98 & 1.03 \\
\hline Ca meq/100 g & 0.47 & 0.26 & 0.23 & 0.32 & 0.32 & 0.24 \\
\hline $\mathrm{K}$ meq $/ 00 \mathrm{~g}$ & 0.03 & 0.03 & 0.04 & 0.03 & 0.04 & 0.04 \\
\hline $\mathrm{Mg}$ meq/100 g & 0.10 & 0.08 & 0.08 & 0.09 & 0.09 & 0.09 \\
\hline $\mathrm{Na}$ meq/100 g & 0.07 & 0.07 & 0.07 & 0.07 & 0.07 & 0.06 \\
\hline $\mathrm{P} \mathrm{mg} / \mathrm{kg}$ & 2.93 & 2.20 & 3.73 & 3.85 & 2.80 & 2.93 \\
\hline $\mathrm{B} \mathrm{mg/kg}$ & 0.23 & 0.20 & 0.18 & 0.15 & 0.18 & 0.15 \\
\hline $\mathrm{Fe} \mathrm{mg} / \mathrm{kg}$ & 23.67 & 22.00 & 34.00 & 38.50 & 71.25 & 87.25 \\
\hline $\mathrm{Cu} \mathrm{mg} / \mathrm{kg}$ & 2.00 & 0.25 & 1.28 & 0.30 & 0.35 & 0.78 \\
\hline $\mathrm{Mn} \mathrm{mg/kg}$ & 1.10 & 1.50 & 1.90 & 13.20 & 11.63 & 14.83 \\
\hline $\mathrm{Zn} \mathrm{mg/kg}$ & 0.57 & 0.18 & 0.58 & 0.33 & 0.25 & 0.35 \\
\hline $\mathrm{S} \mathrm{mg} / \mathrm{kg}$ & 8.67 & 10.00 & 9.25 & 8.50 & 10.00 & 11.25 \\
\hline Acidez & 0.45 & 0.41 & 1.72 & 1.39 & 0.46 & 0.59 \\
\hline Sat. Al & 20.33 & 15.00 & 63.75 & 50.25 & 24.25 & 37.75 \\
\hline
\end{tabular}

Fuente: Banco de datos sobre análisis de suelos. CORPOICA C.I. La libertad

2. Altura de planta: materiales de crecimiento erecto como $A$. gayanus y $V$. zizanioides mostraron mayor desarrollo $(P<0.05)$ con alturas superiores a 115 $\mathrm{cm}$ además de verse favorecidos por la siembra con cepas (material vegetativo). El crecimiento decumbente sobresalió $B$. decumbens siendo su altura $59,8 \%$ y $57,2 \%$ superior $(P<0.05)$ en comparación con $B$. dictyoneura y $B$. humidicola, respectivamente. Entre las leguminosas no se observaron diferencias estadísticas siendo la de mayor altura $P$. phaseoloides $(26 \mathrm{~cm})$, seguida de $S$. capitata $(21.2 \mathrm{~cm}$ ) y finalmente $D$. ovalifolium $(14 \mathrm{~cm})$ (Tabla 2). Aunque el establecimiento inicial de $B$. dictyoneura en comparación con el $B$. 
decumbens y $B$. humidicola, fue más lento, debido al proceso de germinación de la semilla caracterizado por presentar un fenómeno llamado "dormancia", no obstante, una vez alcanzado el óptimo establecimiento las plantas proporcionan una cobertura del $77 \%$, con buen desarrollo estolonifero vigoroso y agresivo. El $B$. humidicola macolla tanto, que a partir de un solo tallo puede crecer hasta cubrir medio metro cuadrado (Argel et al., 2006).

Las mayores $(\mathrm{P}<0.05)$ coberturas las presentaron $D$. ovalifolium $(88.3 \%)$ y las tres especies de Brachiaria: dictyoneura (93.3\%), decumbens $(90.0 \%)$, y humidicola $(76.7 \%)$ aunque este último fue menor $(P<0.05)$ en relación a las demás especies mencionadas (Tabla 2). Igual comportamiento reporta el ICA (1983) en evaluaciones realizadas en la Altillanura encontrando que $S$. capitata presentó una cobertura del $28 \%$ a las 9 semanas de corte.

3. Numero de estolones y longitud: El buen comportamiento agronómico de las especies de Brachiaria estolonoferas quedo demostrado por su mayor numero y longitud de estolones. Según Belalcazar et al, (1995) el crecimiento denso y rizomatoso de las especies permiten un rápido establecimiento, como es el caso de $B$. dictyoneura que desarrolla un gran número de estolones pero de corta longitud a diferencia del B. humidicola (Tabla 2).

4. Producción de forraje seco: el rendimiento de $A$. gayanus $(7.219 \mathrm{~kg} / \mathrm{ha})$ fue superior $(P<0.05)$ con relación a las demás especies, siguiendo en su orden por $V$. zizanioides y $B$. decumbens a los 120 días 5.343 y $3.678 \mathrm{~kg} / \mathrm{ha}$ respectivamente siendo el de menor producción $(\mathrm{P}<0.05)$ D. ovalifolium $(798$ $\mathrm{kg} / \mathrm{ha}$ ). Si se calcula el rendimiento anual de materia seca (MS) en tres cortes de $B$. decumbens sería $11.034,9 \mathrm{~kg} / \mathrm{ha}$ en esta zona de altillanura, cantidad superior, si se compara con la del piedemonte, donde las condiciones de suelo y clima son más favorables puede fluctuar entre 6.000 y $10.000 \mathrm{~kg} / \mathrm{ha} / \mathrm{año}$ cuando se realizan entre 6 y 10 cortes/año, estimando que en época seca los rendimientos de MS, pueden disminuir entre el 30 y 50\%, donde la intensidad de la reducción depende de la duración del periodo de sequía y de las condiciones del suelo (FAO, 2000). Entre las leguminosas, la especie $P$. 
phaseoloides demostró mayor productividad alcanzando con una producción de materia seca de $1.178 \mathrm{~kg} / \mathrm{ha}$ en el primer corte a los 4 meses después de la siembra. Este resultado se puede considerar similar al encontrado en San José del Guaviare, en donde la producción de materia seca en tres frecuencias de corte (6, 9 y 12 semanas) varió entre 5.000 y 6.000 kg/ha por año (Belalcázar et al., 1995).

Tabla 2. Variables agronómicas de praderas en materiales herbáceos

\begin{tabular}{cccccc}
\hline Especie & $\begin{array}{c}\text { Altura } \\
\mathbf{( c m )}\end{array}$ & $\begin{array}{c}\text { Cobertura } \\
\mathbf{( \% )}\end{array}$ & $\begin{array}{c}\text { No } \\
\text { estolones }\end{array}$ & $\begin{array}{c}\text { Long. } \\
\text { Estolones } \\
(\mathbf{c m})\end{array}$ & $\begin{array}{c}\text { Forraje } \\
\mathbf{s e c o} \\
\mathbf{( k g / h a} \text { ) }\end{array}$ \\
\hline A. gayanus & $131.3^{\mathrm{a}}$ & $48.3^{\mathrm{c}}$ & ---- & --- & $7219.0^{\mathrm{a}}$ \\
V. zizanioides & $115.0^{\mathrm{a}}$ & $36.7^{\mathrm{cd}}$ & --- & --- & $5343.0^{\mathrm{ab}}$ \\
B. decumbens & $54.7^{\mathrm{b}}$ & $90.0^{\mathrm{a}}$ & --- & --- & $3678.3^{\mathrm{abc}}$ \\
B. dictyoneura & $32.7^{\mathrm{bc}}$ & $93.3^{\mathrm{a}}$ & $12.7^{\mathrm{a}}$ & $81.3^{\mathrm{ab}}$ & $2471.0^{\mathrm{bc}}$ \\
B. humidicola & $31.3^{\mathrm{bc}}$ & $76.7^{\mathrm{ab}}$ & $9.7^{\mathrm{b}}$ & $90.0^{\mathrm{a}}$ & $1753.7^{\mathrm{bc}}$ \\
P. phaseoloides & $26.0^{\mathrm{bc}}$ & $70.7^{\mathrm{b}}$ & $3.3^{\mathrm{c}}$ & $76.3^{\mathrm{ab}}$ & $1177.7^{\mathrm{c}}$ \\
S. capitata & $21.3^{\mathrm{bc}}$ & $28.3^{\mathrm{d}}$ & ---- & --- & $1077.7^{\mathrm{c}}$ \\
D. ovalifolium & $14.0^{\mathrm{c}}$ & $88.3^{\mathrm{ab}}$ & ---- & --- & $798.0^{\mathrm{c}}$ \\
\hline
\end{tabular}

Promedios seguidos con la misma letra en la columna no presentan diferencia significativa según prueba de Tukey $(\mathrm{P}>0.05)$.

Aunque el $D$. ovalifolium, es la leguminosa de más baja productividad del ensayo $(P<0.05)$ con $708 \mathrm{~kg} / \mathrm{ha}$ después de 120 días, lo cual coincide con lo reportado por Lemus y Lemus, (2004) quienes reportan que la producción de esta especie en condiciones de la altillanura colombiana, fue de $900 \mathrm{~kg} \mathrm{MS} / \mathrm{ha}$ en cortes realizados a las 12 semanas de recuperación de las plantas en época de máxima precipitación y de $800 \mathrm{~kg}$ MS/ha en mínima precipitación, con la misma frecuencia de corte.

5. Evaluación de prácticas de preparación y manejo en suelos descubiertos: El comportamiento de establecimiento de las herbáceas en estos suelos utilizando cuatro sistemas de mecanización fue similar $(P<0.05)$ para las variables: altura de planta oscilando entre 45 y $50.7 \mathrm{~cm}$, cobertura del suelo se observó entre 70 a $74.3 \%$ y número y longitud de estolones que variaron entre 5 a 6.3 talluelos con una longitud que vario entre 49.3 y $66 \mathrm{~cm}$ (Tabla 3). 
Tabla 3. Variables agronómicas de praderas de $B$. decumbens - B. dictyoneura bajo diferentes tratamientos de mecanización

\begin{tabular}{|c|c|c|c|c|c|}
\hline Tratamiento & $\begin{array}{l}\text { Altura } \\
\text { (cm) }\end{array}$ & $\begin{array}{c}\text { Cobertura } \\
(\%)\end{array}$ & $\begin{array}{c}\text { № } \\
\text { estolones }\end{array}$ & $\begin{array}{l}\text { Long. } \\
\text { Estolones } \\
(\mathrm{cm})\end{array}$ & $\begin{array}{c}\text { Biomasa } \\
\text { (kgMS/ha) }\end{array}$ \\
\hline $\begin{array}{c}\mathrm{T}_{1}=1 \text { pase de } \\
\text { rastra + correctivos } \\
\mathrm{T}_{2}=1 \text { pase de }\end{array}$ & 50.7 & 74.3 & 5.3 & 66.0 & 2.904 .5 \\
\hline $\begin{array}{c}\text { rastra + correctivos } \\
+ \text { rastra }\end{array}$ & 45.3 & 71.7 & 6.3 & 60.0 & 1.796 .1 \\
\hline $\begin{array}{c}\mathrm{T}_{3}=2 \text { pases de } \\
\text { rastra }+ \text { correctivos } \\
\mathrm{T}_{4}=1 \text { pase de }\end{array}$ & 48.0 & 70.0 & 5.0 & 49.3 & 2.000 .3 \\
\hline $\begin{array}{c}\text { rastra + correctivos } \\
+ \text { cincel }\end{array}$ & 45.0 & 73.3 & 5.0 & 61.3 & 2.407 .3 \\
\hline Significancia & ns & Ns & ns & Ns & ns \\
\hline $\mathrm{CV}(\mathrm{P}>0.05)$ & 14.3 & 12.1 & 17.7 & 31.4 & 25.47 \\
\hline
\end{tabular}

${ }^{*} \mathrm{~ns}=$ no significativa; $(\mathrm{P}>0.05)$

El tratamiento 1 que corresponde a la preparación de suelo con un pase de rastra sin correctivos, obtuvo los valores más altos de las variables agronómicas y oferta de forraje $2904.5 \mathrm{~kg} \mathrm{MS} / \mathrm{ha}$. Para que el establecimiento de una pradera sea exitoso es necesario considerar aspectos como la preparación del terreno y las estrategias de siembra de las especies adecuadas con el fin de asegurar una cobertura homogénea de los materiales y persistencia de los mismos (Altieri y Nicholls, 2000).

Los resultados de producción de materia seca bajo los cuatro sistemas de labranza oscilo entre 1796 y $2904.5 \mathrm{~kg} \mathrm{MS} /$ ha. Valores inferiores obtuvo Acosta, (1992) en una finca del piedemonte, en suelos de terraza alta, donde evaluó el cincel rígido, cincel vibratorio, arado de discos para recuperar una pradera de $B$. decumbens, después de dos años de evaluación, no se presentaron diferencias significativas en la producción de biomasa, la cual estuvo entre 1193 y $1551 \mathrm{~kg}$ MS/ha, como promedio de cuatro evaluaciones realizadas durante dos años.

En la altillanura colombiana se han realizado diversos trabajos de labranza convencional (dos pases de rastra) comparada con la vertical utilizando cincel vibratorio. Se ha demostrado que para la recuperación de praderas puede 
utilizarse cualquiera de los dos implementos, pues no hay diferencias en el efecto sobre las características físicas de los suelos, ni la producción de forraje (CORPOICA, 2000).

\section{Evaluación de fuentes fosforadas en suelos descubiertos}

Establecimiento y cobertura: las especies $B$. decumbens y $B$. dictyoneura fueron sembradas para conocer la influencia de diferentes fuentes de fosforo comerciales bajo similares dosificaciones de aplicación a niveles entre 5 y 10 ppm de fosforo durante 120 días de establecimiento, al inicio se observaron diferencias significativas $(P<0.05)$, entre los tres tratamientos, los resultados demuestran que la aplicación con DAP (tratamiento 3) incrementó satisfactoriamente la producción de biomasa, observándose una población de plantas homogénea y vigorosa después de la aplicación de la fuente de fósforo soluble. El tratamiento 2 (roca fosfórica), mostró con un proceso de germinación lento y plantas de bajo vigor. La cobertura del suelo con aplicación de DAP permitió obtener un promedio de $88 \%$, mientras que la aplicación de roca fosfórica apenas alcanzó un 35\% (Tabla 4)

Es importante resaltar el comportamiento durante la fase de establecimiento de la pradera con el tratamiento 1 , en donde se aplicó de solubilidad media que complementada con urea, fuente de alta solubilidad permitió un desarrollo uniforme y vigoroso de la pradera muy similar al comportamiento observado con el tratamiento 3 (Tabla 4).

Altura de planta: en los tratamientos con DAP y abono paz del rio sus alturas fueron superiores $(P<0.05)$, alcanzando $57.7 \mathrm{~cm}$ y $56.7 \mathrm{~cm}$ respectivamente, mientras que el de roca fosfórica siempre mantuvo un comportamiento deficiente, plantas de bajo porte $(37.5 \mathrm{~cm})$ y vigor desde el inicio de establecimiento (Tabla 4).

Numero de estolones y longitud: con el tratamiento 3 se alcanzó la mejor respuesta de las plantas con un promedio de 6 estolones por planta con una longitud superior a $110 \mathrm{~cm}$, mientras que con el tratamiento 2 no se encontró 
emisión y desarrollo de talluelos. El comportamiento de las plantas con el tratamiento 1 fue muy similar al observado con el tratamiento 3 , solamente que el número de estolones fue inferior (3.0) (Tabla 4).

Producción de forraje: La mayor producción de materia seca de la mezcla $B$. decumbens-dictyoneura, se alcanzó con la aplicación de DAP (46 kg/ha $\mathrm{P}_{2} \mathrm{O}_{5}$ ) obteniendo $3080 \mathrm{~kg} \mathrm{MS} / \mathrm{ha}$, seguido por el tratamiento con abono paz del rio y finalmente con roca fosfórica $960 \mathrm{~kg}$ MS/ha (Tabla 4).

Tabla 4. Variables agronómicas de B. decumbens-dictyoneura con diferentes fuentes de fosforo durante la fase de establecimiento.

\begin{tabular}{cccccc}
\hline Tratamiento & $\begin{array}{c}\text { Altura } \\
(\mathbf{c m})\end{array}$ & $\begin{array}{c}\text { Cobertura } \\
(\%)\end{array}$ & $\begin{array}{c}\text { No } \\
\text { estolones }\end{array}$ & $\begin{array}{c}\text { Long. } \\
\text { Estolones } \\
(\mathbf{c m})\end{array}$ & $\begin{array}{c}\text { Biomasa } \\
\text { (kg } \\
\text { MS/ha) }\end{array}$ \\
\hline $\begin{array}{c}\text { Abono paz del rio } \\
\left(46 \mathrm{~kg} / \mathrm{ha}_{2} \mathrm{O}_{5}\right)\end{array}$ & $56.7^{\mathrm{a}}$ & 55.0 & $3.0^{\mathrm{ab}}$ & $95.3^{\mathrm{a}}$ & $2.800 .0^{\mathrm{a}}$ \\
$\begin{array}{c}\text { Roca fosfórica } \\
\left(46 \mathrm{~kg} / \mathrm{ha} \mathrm{P}_{2} \mathrm{O}_{5}\right)\end{array}$ & $27.0^{\mathrm{b}}$ & 35.7 & $0.0^{\mathrm{b}}$ & $0.0^{\mathrm{b}}$ & $960.0^{\mathrm{b}}$ \\
DAP $\left(46 \mathrm{~kg} / \mathrm{ha}_{2} \mathrm{O}_{5}\right)$ & $57.7^{\mathrm{a}}$ & 88.3 & $6.3^{\mathrm{a}}$ & $110.0^{\mathrm{a}}$ & $3.080 .0^{\mathrm{a}}$ \\
\hline
\end{tabular}

Medias seguidas con la misma letra en la columna no presentan diferencia significativa según prueba de Tukey $(\mathrm{P}>0.05)$

Con base en los resultados observados durante la fase de establecimiento, se pudo constatar que la mejor fuente de fosforo aplicada $(\mathrm{P}<0.05)$ bajo las condiciones típicas de la zona fue el DAP (46 kg/ha $\mathrm{P}_{2} \mathrm{O}_{5}$ ), seguido del abono paz del rio y por último la roca fosfórica, lo cual se basó en la disponibilidad de minerales esenciales para el buen desarrollo de las especies de $B$. decumbens y $B$. dictyoneura adaptadas a condiciones de la altillanura (Salinas y García, 1985). Sin embargo, es importante considerar el costo por unidad de producto y su residualidad en el suelo para asegurar el aporte del nutriente requerido por las plantas, teniendo en cuenta que el fosforo es el nutrimento más limitante para el adecuado desarrollo de las plantas en suelos ácidos (Cánepa et al., 2015).

Teniendo en cuenta que la fertilización de las parcelas experimentales se realizó a una saturación de bases entre 35 y 50\% y niveles de fósforo entre 5 y 10 ppm; la mayor parte de los suelos de la altillanura y de las terrazas del 
piedemonte, se encuentran en un nivel bajo y medio de fertilidad, presentándose alta deficiencia de todos los nutrientes esenciales para el desarrollo de los pastos. La saturación de sus bases en el suelo es menor de $30 \%$ resultando ser limitante para obtener buena respuesta en producción de forraje, además según estudios hechos en suelos ácidos del cerrado brasileño, los pastos como el $B$. decumbens, $B$. humidicola y $B$. dictyoneura exigen una saturación de bases de $30-35 \%$, mientras que en especies más exigentes necesitan 40 a 45\% (Lemus y Lemus, 2004)

El fosforo aportado por el DAP, en un $100 \%$ es asimilable por las plantas, ya que más del $95 \%$ se disuelve en agua y pasa directamente a la solución del suelo, permitiendo su rápida absorción por las plantas, asegurando plantas más vigorosas de mejor crecimiento y desarrollo de macollas y estolones.

Evaluación de materiales arbustivos: emergencia y sobrevivencia, teniendo en cuenta que los materiales arbustivos presentaron una germinación poco uniforme fue necesario programar una resiembra de plantas a inicios de la temporada de lluvias. Para tal fin se dispuso la producción de plantas en vivero y luego se realizó la evaluación de la fase de establecimiento. Aunque las especies seleccionadas presentan buena adaptabilidad a suelos ácidos de baja fertilidad, son materiales de crecimiento lento durante los primeros meses de establecimiento. Según Argel et al., (2006), las especies arbustivas pueden producir más biomasa que las herbáceas, toleran mejor el mal manejo y tiene la capacidad de rebrotar y ofrecer forraje de buena calidad en zonas con sequias prolongadas.

Evaluación de materiales arbóreos: Una vez transcurridos los primeros dos meses desde la siembra de las especies arbóreas se realizó una evaluación que incluyó variables como diámetro a la altura del pecho (D.A.P), altura total, y mortalidad. Además, se estudió el vigor de las plantas que indica su estado general y su respuesta frente a las condiciones del área de siembra, para esto se utilizó una escala de uno (1) a cinco (5), en donde 5 corresponde a excelente respuesta y estado general y 1 es un árbol muerto o afectado por 
alguna plaga o enfermedad (también incluye daños mecánicos por agentes externos) (Tabla 5).

Tabla 5. Variables dasométricas de especies arbóreas en condiciones de serranía durante la fase de establecimiento

\begin{tabular}{ccccc}
\hline Especie & $\begin{array}{c}\text { Altura } \\
(\mathbf{c m})\end{array}$ & $\begin{array}{c}\text { Diámetro } \\
(\mathbf{c m})\end{array}$ & $\begin{array}{c}\text { Vigor } \\
(\mathbf{1 - 5})\end{array}$ & $\begin{array}{c}\text { Mortalidad } \\
(\%)\end{array}$ \\
\hline Acacia mangium & 68 & 1 & 5 & 1 \\
Anacardium occidentale & 28 & 1 & 3 & 1 \\
Piptadenia opacifolia & 38 & 2 & 4 & 4 \\
Eucalipthus pellita & 69 & 1 & 5 & 6 \\
Simarouba amara & 38 & 0.5 & 4 & 3 \\
Pinus caribeae & 7 & 0.5 & 1 & 7 \\
\hline
\end{tabular}

Las especies $E$. pellita y $A$. mangium son las que en términos generales mostraron un mejor comportamiento en cuanto a crecimiento superadas solo en diámetro por la especie $P$. opacifolia. El comportamiento de la especie $P$. caribeae corresponde más a daños ocasionados por transporte y manipulación que a la respuesta a las condiciones propias del sitio. Es muy interesante el comportamiento de las especies $P$. opacifolia y $S$. amara las cuales han sido poco estudiadas en este tipo de suelo. Su desarrollo inicial supera los valores de estas mismas especies en arboretum lo que indica su respuesta a variables como manejo de suelos y fertilización.

La mortalidad y el vigor corresponden a valores muy por debajo de los manejados en plantaciones con fines de aprovechamiento y protección de ecosistemas. Los daños por insectos son ocasionales y han sido bien controlados. Dentro de este ensayo se incluyó la siembra de las especies herbáceas $D$. ovalifolium, $P$. phaseoloides y $S$. capitata como cobertura.

\section{CONCLUSIONES Y RECOMENDACIONES}

En la evaluación de materiales herbáceos, las especies de gramíneas principalmente del genero Brachiaria, demostraron buena adaptabilidad y desarrollo en suelos ácidos de baja fertilidad con rápido establecimiento y buena cobertura del suelo. 

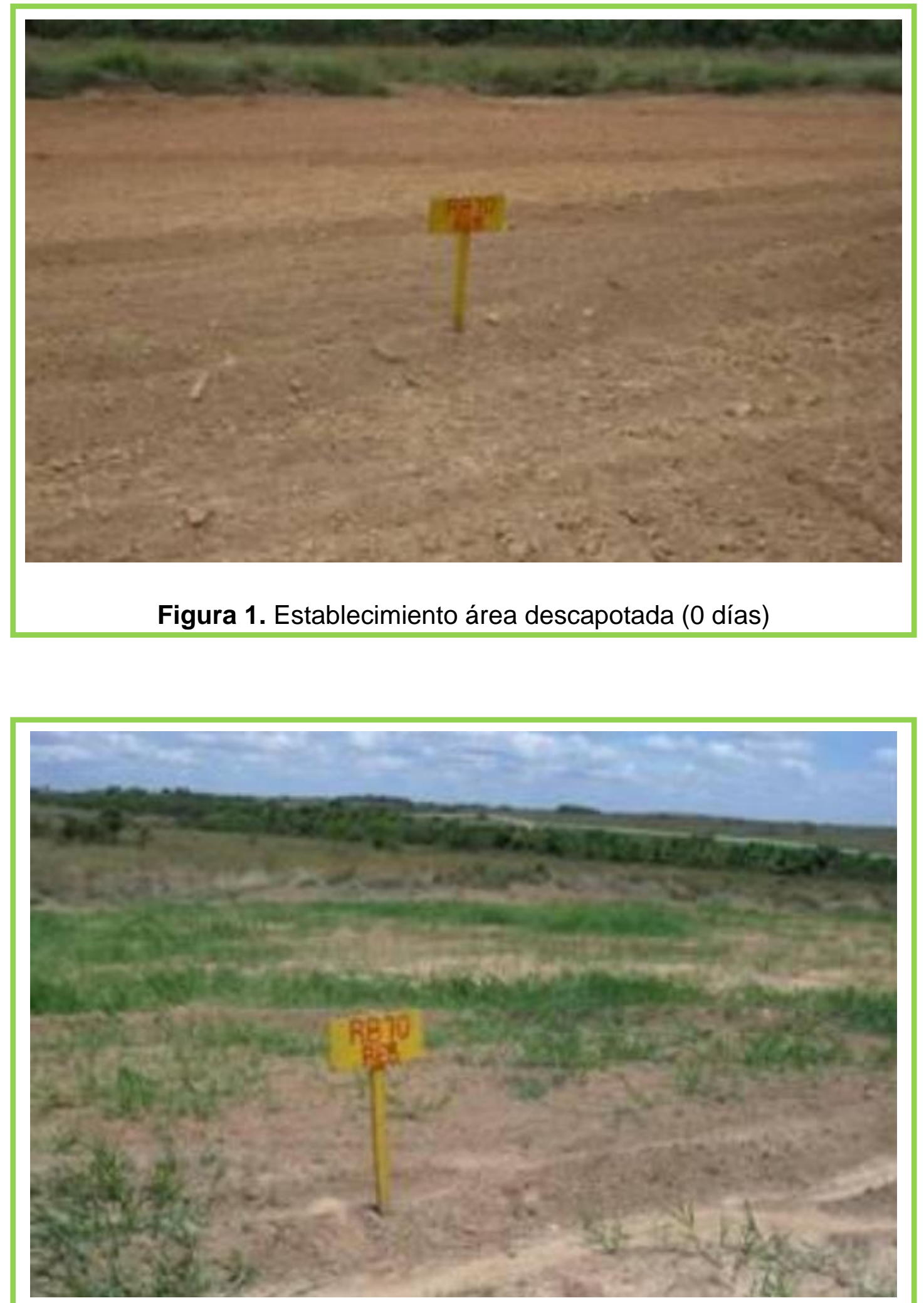

Figura 2. Evaluación de emergencia área descapotada (20 días) 


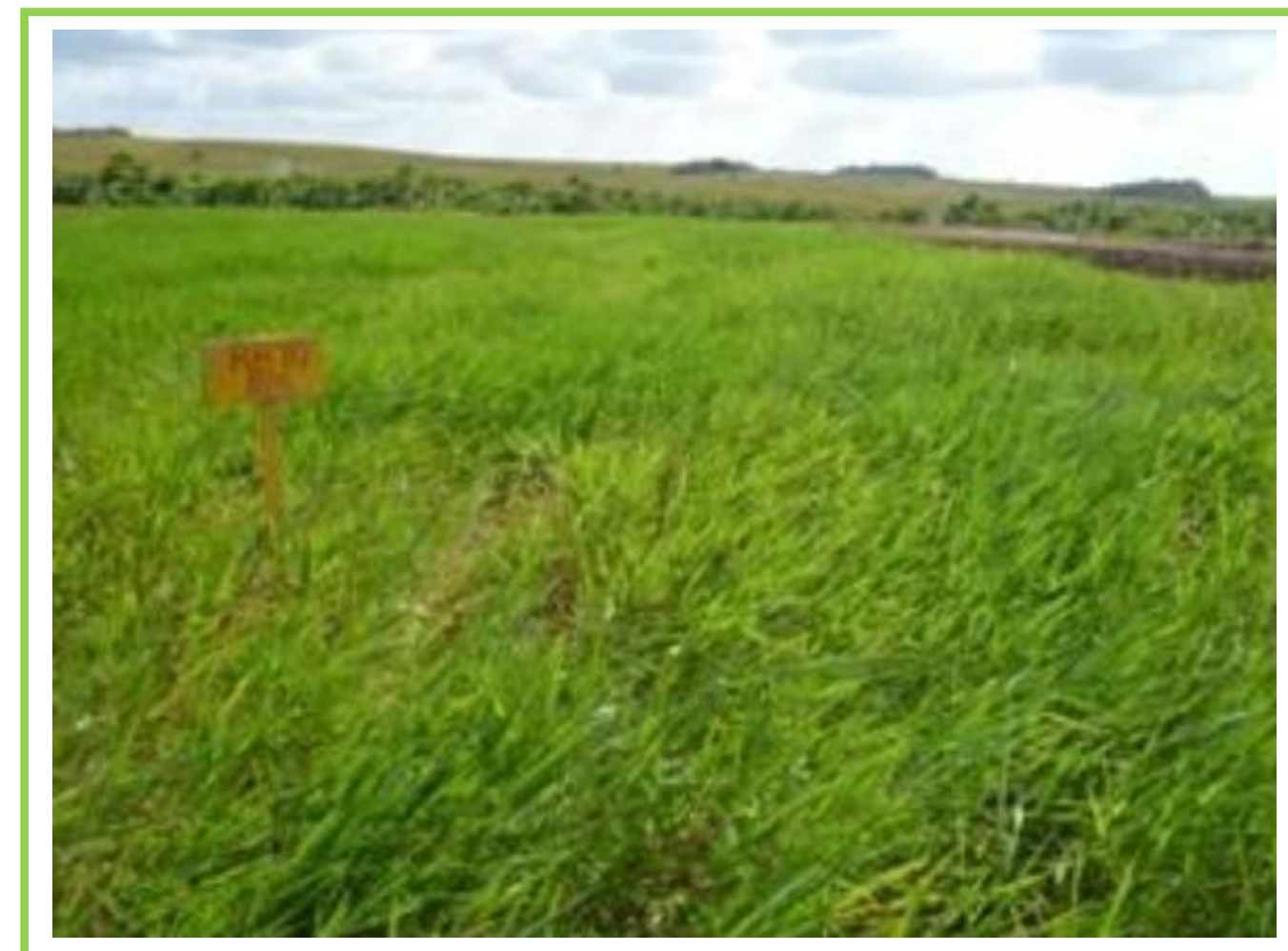

Figura 3. Evaluación de cobertura 95\% área descapotada (120 días)

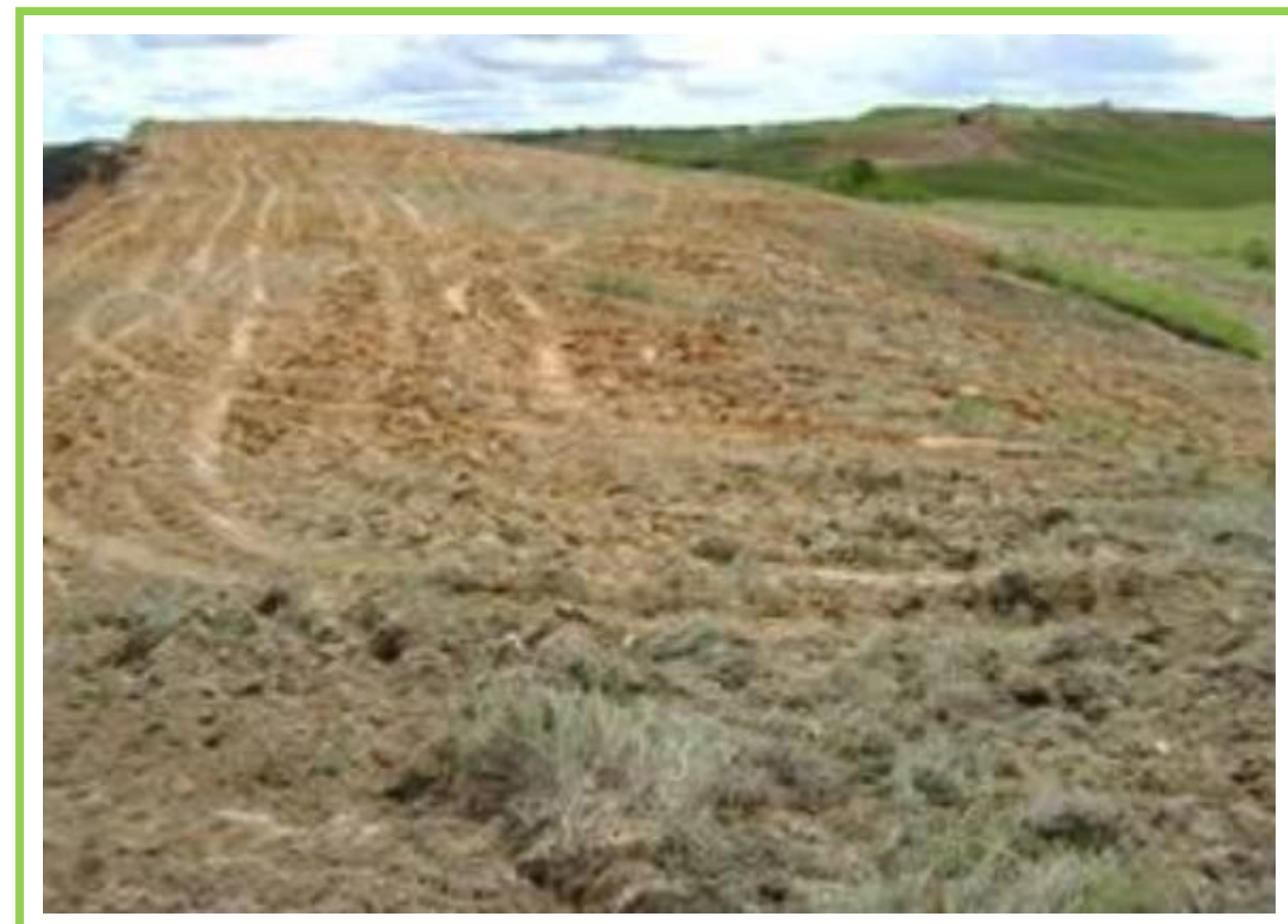

Figura 4. Establecimiento área con plintita expuesta (0 días) 


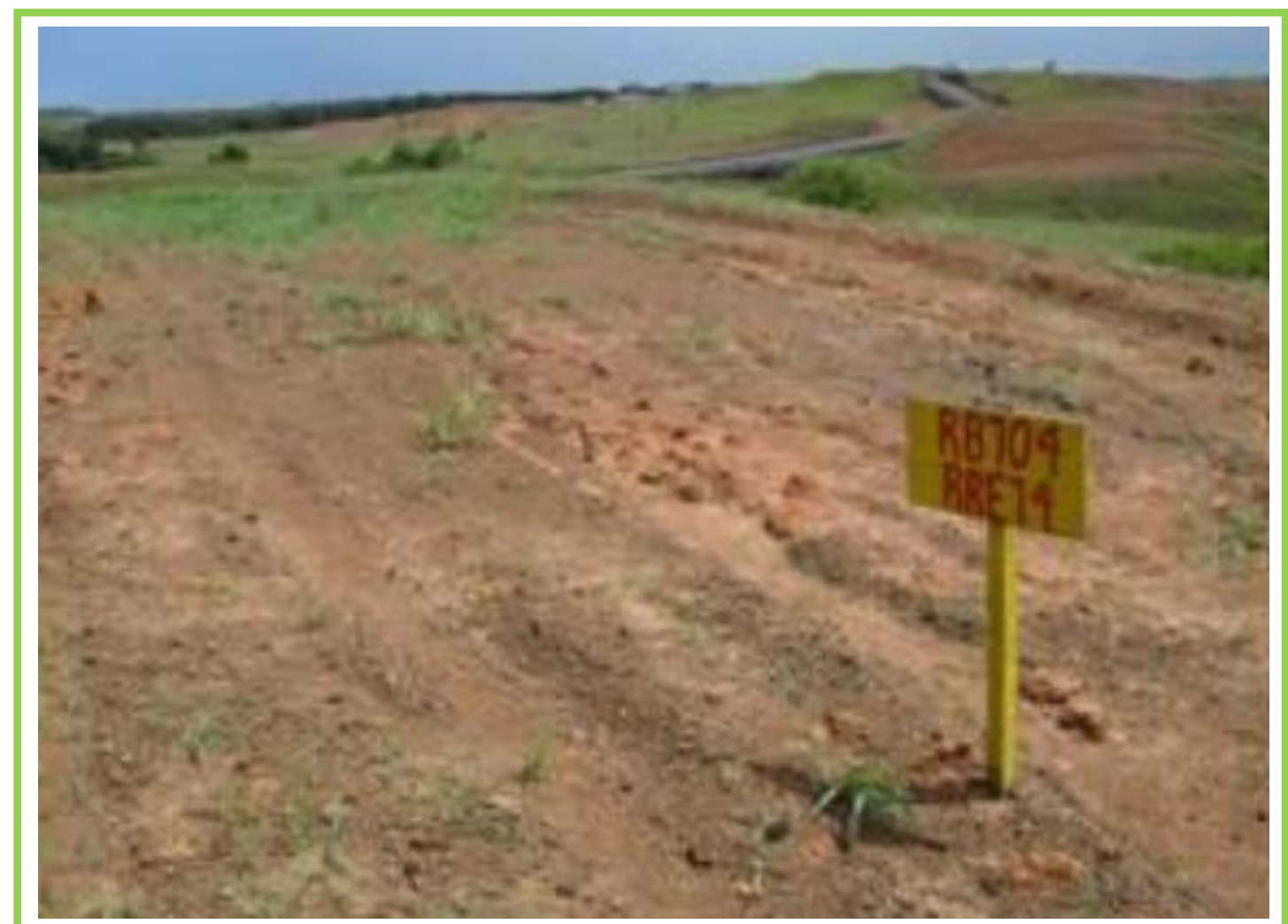

Figura 5. Evaluación de emergencia área con plintita expuesta (20 días)

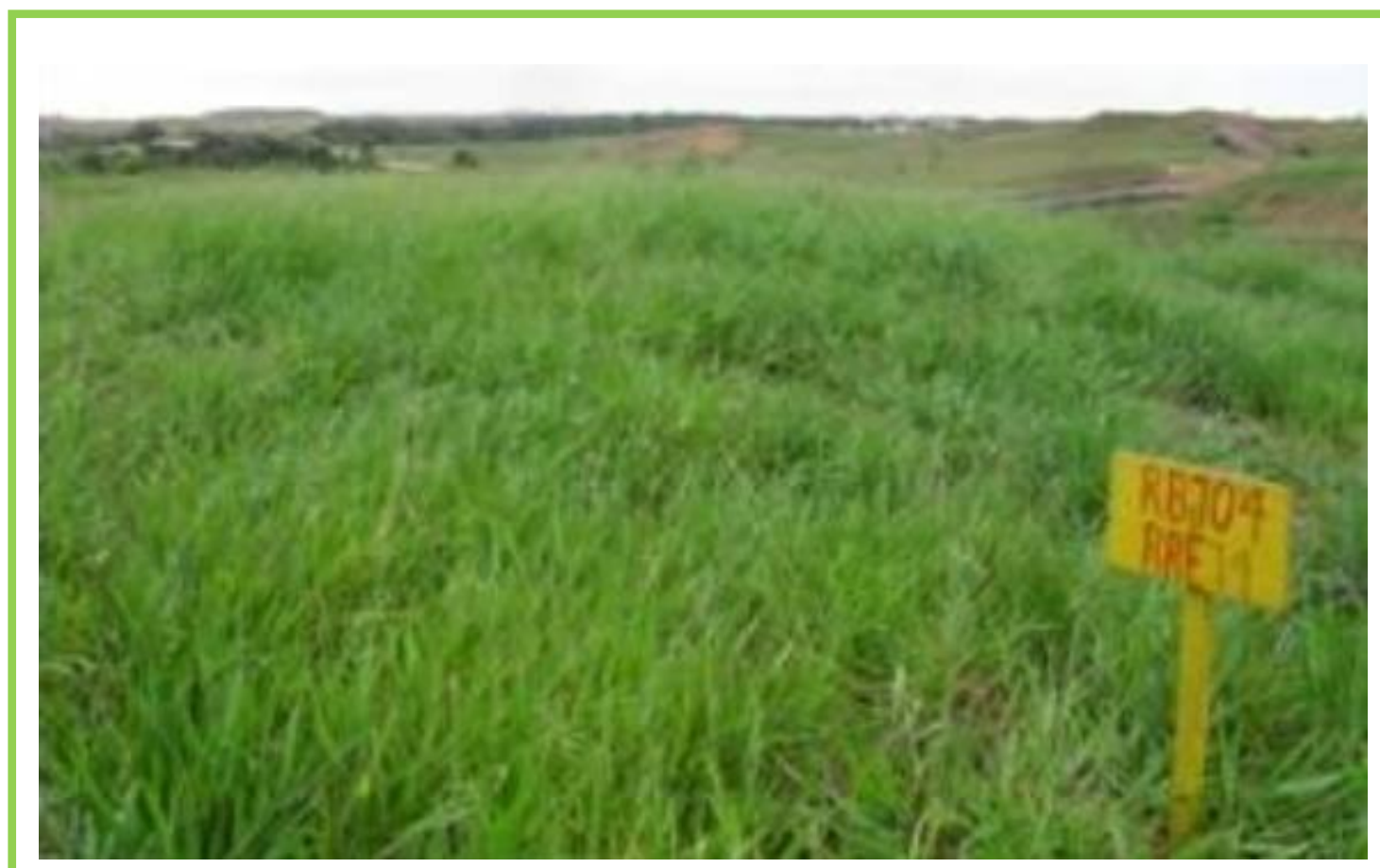

Figura 6. Evaluación de cobertura con plintita expuesta 95\% (120 días) 
En relación con la producción de biomasa los materiales establecidos con material vegetativo $A$. gayanus y $V$. zizanioides alcanzaron los rendimientos más altos.

Aunque la respuesta a los tratamientos de preparación, aplicación de correctivos y siembra no arrojo diferencias importantes para las variables evaluadas, se destacó el tratamiento 1 por las facilidades y bajos costos que representa para establecer praderas en condiciones de la altillanura disectada. No obstante, la mejor respuesta en términos agronómicos se observó con la aplicación de DAP como fuente fosforada.

En fases muy tempranas del cultivo se destacan las especies E. pellita, $A$. mangium y $P$. opacifolia por su buen comportamiento y adaptabilidad reflejado en su crecimiento y vigor en condiciones en clima y suelos de la serranía.

\section{REFERENCIAS BIBLIOGRÁFICAS}

1. Acosta A. Evaluación agronómica de métodos de renovación de praderas de Brachiaria decumbens en el Piedemonte Llanero. Instituto Colombiano Agropecuario - ICA, Villavicencio, Meta. 1992.

2. Altieri M, Nicholls $C$. Agroecología, Teoría y práctica para una agricultura sustentable. Programa de las Naciones Unidas para el Medio Ambiente, Red de Formación Ambiental para América Latina y el Caribe. México DF, México, 250 p. 2000.

3. Argel PJ, Miles JW, Guiot JD, Lascano CE. Cultivar Mulato (Brachiaria hybrid CIAT 36061): A high yielding, high-quality forage grass for the tropics. Centro Internacional de Agricultura Tropical (CIAT): Cali, Colombia, 24 p. 2006.

4. Belalcázar DJ, Lemus LH, Durán CV. Especies forrajeras tropicales de interés para pasturas en suelos ácidos de Colombia. Fascículo 2. Capacitación en tecnología de producción de pastos. Centro Internacional de Agricultura Tropical (CIAT). Cali, Colombia. 1995.

5. Burbano G. Alternativas ambientales para mitigar la erosión en la microcuenca la cofradía del municipio de San Francisco Putumayo. Trabajo de Grado Especialista en Gerencia Ambiental, Escuela Superior de Administración Pública. Sibundoy Putumayo, 61 p. 2007.

6. Cánepa Y, Trémols AJ, González A, Hernández A. Situación actual de los suelos tabacaleros de la empresa Lázaro Peña de la provincia Artemisa. Cultivos Tropicales, 36 (1): 80-85. 2015.

7. Castañeda O, Contreras F. Ecosistemas costeros mexicanos una actualización, Universidad Autónoma Metropolitana Unidad Iztapalapa, México. 2003. Disponible En: http://investigacion.izt.uam.mx/ocl/mapa.html

8. CIAT. Establecimiento y renovación de pasturas. Memorias VI Reunión del Comité asesor de la RIEPT. Veracruz, México 425 p. 1988. 
9. CIAT. Manejo y utilización de pasturas en suelos ácidos de colombia. en: unidades de aprendizaje para la capacitación en tecnología de producción de pastos. 1994.

10. CORPOICA (Corporación Colombiana de Investigación Agropecuaria). Cartilla "Alimentación Bovina". Ed Gómez J, Centro de Investigación Palmira, 59 p. 2000.

11. FAO (Organización de las Naciones Unidas para la Alimentación y la Agricultura). Los principales factores ambientales y de suelos que influyen sobre la productividad y el manejo. 7 p. 2000. Disponible En: http://mazinger.sisib.uchile.cl/repositorio/ap/ciencias agronomicas/c20021221 046edafo factoresambientalesysuelos.pdf

12. Lemus $L$, Lemus V. Plantas de uso forrajero en el trópico cálido y templado de Colombia, Universidad de los Llanos, Programa de Ingeniería Agronómica, Villavicencio, Meta, Colombia. 360 p. 2004.

13. León JD. Estudio y control de la erosión hídrica. Universidad Nacional de Colombia, Facultad de Ciencias Agropecuarias, Departamento de Ciencias Forestales, Centro de Publicaciones, Sede Medellín, 224 p. 2001.

14. Navarro GH, Pérez OMA, Castillo GF. Evaluación de cinco especies vegetales como cultivos de cobertura en valles altos de México. Revista Fitotecnia Mexicana, 30 (2): 151-157. 2007.

15. Pérez BR, Cuesta MP. Especies Forrajeras para el Piedemonte Llanero. 1992. Manejo y Producción Animal. En: Pastos y Forrajes para Colombia. Suplemento Ganadero. ICA Banco Ganadero, p 85-94. 1992.

16. Pérez BR, Rincón A, Bueno G, Vargas O, Cuesta P. Alternativas de establecimiento de praderas. Octubre 2000. Bogotá. Corporación Colombiana de Investigación Agropecuaria, CORPOICA. Innovación y Cambio Tecnológico, 1 (2): 56-61. 2000.

17. Pérez LO, Pérez BR. Informe final proyecto Evaluación agronómica y productiva de especies forrajeras en la Orinoquia Colombiana. CORPOICA. C.I. La Libertad. Villavicencio. Meta. 45 p. 2003.

18. Rincón A. Degradación y Recuperación de Praderas en los Llanos Orientales de Colombia. Boletín Técnico № 19. CORPOICA - PRONATTA. Villavicencio. Meta. Colombia. 48 p. 1999.

19. Rincón A, Cuesta P, Pérez R, Bueno G, Pardo O, Gómez J. Manual técnico Producción y utilización de recursos forrajeros en sistemas de producción bovina de la Orinoquia y el Piedemonte Caqueteño. CORPOICA-FEDEGANMADR. Bogotá, Colombia. 76 p. 2002.

20. Teasdale JR. Principios y prácticas para el uso de cultivos de cobertura en el manejo de sistemas de malezas. En: Manejo de malezas para países en desarrollo (Addendum I). R. Labrada (ed) FAO - ONU. 2004. Disponible En: http://www.fao.org/3/y5031s/y5031s0j.htm 